\title{
Exact Solution of a Test Particle in Presence of Thick Domain Walls
}

\author{
A. Al-Badawi \\ Physics Department, Al-Hussein Bin Talal University, Ma'an. Jordan
}

\begin{abstract}
We have obtained the exact solution of the equations of motion of a test particle near a thick domain walls for the case of Ricci tensor $\mathrm{R}_{\mathrm{ab}}=0$. From the solution it has been shown that the domain walls have repulsive gravitational fields.
\end{abstract}

Key words: Classical general relativity, Thick domain walls, Behavior of Test particle, Repulsive properties.

\section{INTRODUCTION}

Properties of domain wall have been object of intense investigation for different reasons. One is that domains walls are objects formed in the early stages of the evolution of the universe ${ }^{[1]}$ and have been studied intensively in the past decade or so, this is due to their implications to cosmology ${ }^{[2]}$. Other reason is that the study of topological defects has wide applicability in many areas of physics. In the cosmological area, defects have been put forward as a possible mechanism for structure formation ${ }^{[3]}$. Domain walls are considered the most simple to study in the field of topological defects. They correspond to solutions in one-to-one dimensions, which are extended in two spatial directions to form a wall structure. This is because they depend on only the distance from the wall. The thick domain walls are solutions to Einstein's gravity theory interaction with a scalar field, where the scalar field is a standard topological kink interpolating between the minima of a potential with spontaneously broken symmetry.

Domain walls are formed whenever a discrete symmetry is broken. For example in the Higg's scalar field $\Phi$ with an effective energy potential $V(\Phi, T)$ given by

$V(\Phi, \mathrm{T})=-\mu^{2} \frac{\Phi^{2}}{2}+\lambda \frac{\Phi^{4}}{4}+\left(3 \lambda \Phi^{2}-\mu^{2}\right) \frac{T^{2}}{24}-\pi^{2} \frac{T^{4}}{90}$ where $\lambda$ is the coupling constant, $\mathrm{T}$ the temperature of the universe, and $\mu$ a real constant ${ }^{[4]}$. We can determine the equilibrium values of $\Phi$ from

$$
\frac{d V(\Phi, T)}{d \Phi}=\lambda \Phi\left[\Phi^{2}-\frac{T c^{2}-T^{2}}{4}\right]=0,
$$

where $T_{c}=\frac{2 \mu}{\sqrt{\lambda}}$

If $\mathrm{T}>\mathrm{T}_{\mathrm{c}}$, symmetry is resorted, since $\mathrm{V}(, \Phi, \mathrm{T})$ has only one minimum. Therefore the universe becomes colder, when $\mathrm{T}$ becomes cooler than $\mathrm{Tc}$, then the Higges scalar field $\Phi$ acquires two other non-zero exception values which implies that we have two regions $\langle\Phi\rangle_{+}$and $\langle\Phi\rangle_{-}$. If one goes from $\langle\Phi\rangle_{+}$to \langle $\Phi\rangle_{\text {., }}$ one should pass through the unstable region $\langle\Phi\rangle$ $=0$. Therefore, in between the regions there exist an energy layer with a surface energy density $\sigma$. This layer is called domain wall.

Many authors ${ }^{[5,6]}$ have discussed non-static solutions of the Einstein scalar field equations for thick domain wall. In these solutions the energy scalar is independent of time while the metric tensor depends on both space and time. Recently, Wang ${ }^{[7]}$ obtained a class of solutions to the Einstein's equations representing the gravitational collapse of a thick domain wall. We can consider the domain wall as an interesting gravitational object. Its metric is not static but time dependent ${ }^{[8,9]}$, having a de sitter-like expansion in the plane of the wall. Observers experience repulsion from the domain wall, and there is a horizon at finite proper distance from the defect's core. This horizon can be interpreted as a facet of the choice of coordinates, which usually use the flat space wall solution as a starting point, and impose planer symmetry on the domain wall spacetime. However in Ref. ${ }^{[10,11]}$ they have use different set of coordinates such that the wall has the appearance of a bubble which counteracts in from infinite radius to some minimum radius, and then re-expand undergoing uniform acceleration from the origin. The horizon is then simple the light core of the origin in these coordinates, and is somewhat similar to the horizon of rindle space-time.

In this study we have obtained the exact solution of the geodesies equation of motion of a test particle in the presence of the thick domain wall for the case of vacuum solution. The solution implies that the domain wall have repulsive gravitational field.

Formulation and Calculations: Let us consider the general metric for a plane symmetric space-time as follow

$$
d s^{2}=e^{v(x, t)} d t^{2}-e^{\lambda(x, t)} d z^{2}-e^{\psi(z, t)}\left(d x^{2}+d y^{2}\right)
$$

This metric have three killing vectors namely

$\delta_{\mathrm{x}}, \delta_{\mathrm{y}}, \mathrm{x} \delta_{\mathrm{y}}-\mathrm{y} \delta_{\mathrm{x}}$

Assume that the scalar field $\Phi$ a function of $\mathrm{z}$ only. Then the components of the energy momentum tensor

$T_{\mu v}=\frac{\partial^{2} \Phi}{\partial_{\mu} \partial_{v}}-g_{\mu v}\left(\frac{1}{2} \partial_{\alpha} \Phi \partial^{\alpha} \Phi-V(\Phi)\right)$ 
is given by,

$T_{t}^{t}=T_{x}^{x}=T_{y}^{y}=\frac{1}{2} \bar{e}^{\lambda} \Phi^{2}+v(\Phi)=\rho$

$T_{z}^{z}=-\frac{1}{2} \bar{e}^{\lambda} \Phi^{2}+V(\Phi)=-\rho$

Where the scalar field equation becomes

$\Phi^{\prime \prime}+\Phi^{\prime}\left(\dot{\Psi}^{\prime}+\frac{1}{2}\left(v^{\prime}-\lambda^{\prime}\right)\right)=e^{\lambda} \frac{d V(\Phi)}{d \Phi}$

Where "prime and "dot" denote derivatives with respect to $\mathrm{z}$ and $\mathrm{t}$ respectively. Since $\dot{\Phi}=0$, then it implies

$$
\lambda=\lambda(z), \quad \partial_{t}\left(\Psi^{\prime}+\frac{1}{2}\left(v^{\prime}-\lambda^{\prime}\right)=0 .\right.
$$

And

$$
G_{t}^{z}=0 \Rightarrow 2 \dot{\Psi}^{\prime}-v^{\prime} \dot{\Psi}+\dot{\Psi} \Psi^{\prime}=0
$$

This puts the line element (1) in the form

$$
d s^{2}=A(z)\left[d t^{2}-d z^{2}-b(t)\left(d x^{2}+d y^{2}\right)\right]
$$

From the remaining three Einstein equations we can determine the functions $\mathrm{A}(\mathrm{z}), \mathrm{b}(\mathrm{t})$, and $\Phi(\mathrm{z})$ namely

$$
\begin{aligned}
& G_{t}^{t}-G_{x}^{x}=0 \Rightarrow \ddot{b} b-\dot{b}^{2}=0 \\
& G_{x}^{x}-G_{z}^{z}=-\frac{A^{\prime \prime}}{A^{2}}+\frac{3}{2} \frac{A^{\prime 2}}{A^{3}}-\frac{C^{2}}{2 A}=8 \pi G \frac{1}{A} \Phi^{\prime 2} \\
& G_{t}^{t}+G_{z}^{z}=-\frac{A^{\prime \prime}}{A^{2}}+\frac{C^{2}}{A}=16 \pi G V(\Phi)
\end{aligned}
$$

Where $\mathrm{C}$ is the integration constant. Solution of equation (9) is

$$
\mathrm{b}=\mathrm{e}^{\mathrm{ct}}
$$

From equation's (10) and (11) we can determine A (z) and $\Phi(\mathrm{z})$ for a given $\mathrm{V}(\Phi)^{[5]}$. For the case of Ricci tensor $\mathrm{R}_{\mathrm{ab}}=0$ we will solve equation's (10) and (11). After some mathematical manipulations, equations (10) and (11) reduces to, ${ }^{[10]}$,

$\ddot{u}+\frac{2}{3} \frac{\dot{u}^{2}}{u}-\frac{b^{2}}{3} u^{-\frac{5}{3}}=0$

Where $\quad \mathrm{u}=\mathrm{bz}+1$, The above equation (13) represents the equation of motion of a test particle near a thick domain wall. The exact solution of eq. (13) is obtained which is giving by

$\dot{u}=b u^{-2 / 3} \sqrt{u^{2 / 3}+c}$

Integrating eq. (14) we obtain

$$
\begin{aligned}
& C_{o}+\frac{8 b}{3} t=\left[2 u-3 c u^{1 / 3}\right] \sqrt{u^{2 / 3}+c}+3 c^{2} \\
& \ln \left[u^{1 / 3}+\sqrt{u^{2 / 3}+c}\right]
\end{aligned}
$$

where $\mathrm{c}$ and $\mathrm{C}_{\mathrm{o}}$ are integration constants.

Let us now choose our initial conditions as follows:

At $\mathrm{t}=0, \mathrm{u}=\mathrm{u}_{\mathrm{o}}$ and $\dot{u}=v_{o}$

Imposing equations (16) into equations (14) and (15) we obtain

$$
\begin{aligned}
& C_{o}=v_{o} u_{o}^{5 / 3}\left[5-3 v_{o}^{2} u_{o}^{2 / 3}\right]+3 c^{2} \\
& \ln \left[u_{0}{ }^{1 / 3}+u_{0}{ }^{2 / 3} v_{0}\right] \\
& c=u_{o}{ }^{2 / 3}\left[v_{o}^{2} u_{o}^{2 / 3}-1\right]
\end{aligned}
$$

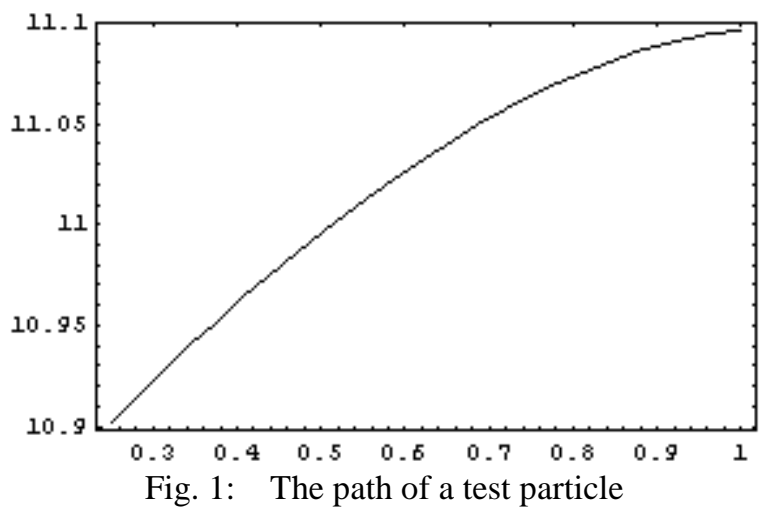

The Fig. 1 (horizontal axis $=\mathrm{z}$ and vertical axis $=\mathrm{t}$ ) represents the path of a test particle near a thick domain wall for the values $\left(\mathrm{v}_{0}=0.6\right.$ and $\left.\mathrm{u}_{0}=10\right)$. If we release the particle at $u=0.6$ (or any value) then it is observed that the particle repels away from the domain wall. This result emphasis that the domain walls have repulsive gravitational fields as had been anticipated by Vilenkinp ${ }^{[12]}$. We should notice that the shape of the figure dose not change when we alter the values of $\left(\mathrm{v}_{0}\right.$ and $\mathrm{u}_{0}$ ).

\section{CONCLUSION}

Thick domain walls are solutions of the coupled Einstein -scalar field equations with Kinklike scalar field distribution and vanishing energy momentum tensor far away from the center of the wave. Exact solution of the equation of motion of a test particle near a thick domain walls is given. We showed that when the particle is released at an initial distance $\mathrm{u}_{0}$ from the domain wall then the particle is repelled far away which proves that the gravitational field of the domain wall has a repulsive properties.

\section{REFERENCES}

1. Kibble, T.W.B., 1976 "Topology of cosmic domains and strings" J. Phys., A9: 1387.

2. Vilenkin, A.,1985 " Nonsingular static global string" Phys. Rep., 121,263; Phys. Rev. Lett., 1994., 72: 3137.

3. Vilenkin, A. and E.P.S. shellard, 1994. Cosmic Strings and other Topological Defect. Cambridge University Press.

4. Linde, A.D., 1979 " Phase transitions in gauge theories and cosmology” Rep. Prog. Phys., 42: 389.

5. Goets, G., 1990 " The gravitational fields of plane symmetric thick domain walls" J. Math. Phys., 31: 2683.

6. Mukheree, M., 1993 "Gravitational fields of cosmic membranes" Class. Quan. Grav. 10: 131.

7. Wang, A., 1994 " Gravitational collapse of thick domain walls" Mod. Phys. Lett., 39: 3605.

8. Widrow, L.M., 1989 “ General-relativistic domain walls” Phys. Rev. D, 39: 3571.

9. Hill, C.T., D.N. Schramm and J.N. Fry, 1989. "Cosmological structure formation from soft topological defects" Comm. Nucl. Part. Phys., 15: 25.

10. Ipser, J. and P. Sikivie, 1984 " Gravitationally repulsive domain wall” Phys.Rev.D, 30: 712.

11. Gibbons, G.W., 1993 " Global structure of supergravity domain wall space-time" Nucl.Phys., B 394: 3-20.

12. Vilenkin, A., 1981 "Gravitational field of vaccum domain walls and strings" Phys. Rev. D, 23: 852. 